\title{
A qualidade da dieta está associada com a ingestão de água em residentes de São Paulo, Brasil
}

\author{
Quality of diet is associated with water intake among residents \\ in São Paulo, Brazil
}

Isabella Lucchesi (https://orcid.org/0000-0001-6750-2767) ${ }^{1}$

Regina Mara Fisberg (https://orcid.org/0000-0002-4490-9035) ${ }^{1}$

Cristiane Hermes Sales (https://orcid.org/0000-0001-8474-2439) ${ }^{1}$

${ }^{1}$ Departamento de

Nutrição, Faculdade de Saúde Pública, Universidade de São Paulo. Av. Dr. Arnaldo 715, Cerqueira César. 01246-904 São Paulo SP Brasil.

cristianehsales@gmail.com

\begin{abstract}
The scope of the study was to evaluate the probability of the adequacy of water intake among residents of São Paulo, and to investigate if the quality of diet differs according to the adequacy of water intake. Data were extracted from the Health Survey of São Paulo, a cross-sectional, population-based study. Water intake (drinking water beverages and food) was evaluated using two 24-hours dietary recalls and a supplementary dietary intake questionnaire. Adequacy and classification of water intake was assessed using the corresponding Adequate Intake for sex and age of the individual. Dietary quality was evaluated using the revised Brazilian Healthy Eating Index (BHEI). The associations were tested using the Theil-Sen median test and logistic regression models. A low probability of adequate water intake was observed, and this decreased with advancing age. Except among the elderly, among whom no association was observed, a positive association between water intake and the BHEI was observed, and for the total and integral fruit components, which was maintained after confusion adjustments. Among adults, it was observed that those who ingested less water had even lower scores for saturated fats. The residents of São Paulo have low water intake, and the quality of diet is worse among those who drink less water.
\end{abstract}

Resumo O objetivo foi avaliar se a qualidade da dieta varia de acordo com a adequação da ingestão de água em residentes de São Paulo. Trata-se do Inquérito de Saúde de São Paulo, estudo transversal de base populacional. A ingestão de água total (potável, de bebidas e de alimentos) foi avaliada por meio de dois recordatórios alimentares de $24 \mathrm{~h}$ e questionário complementar sobre a dieta, e a qualidade da dieta foi avaliada por meio do indice de qualidade da dieta revisado (IQDR) para a população brasileira. A Adequate Intake foi usada para estratificar os indivíduos. As associações foram testadas pelo teste Theil-Sen e pelo modelo de regressão logística. Observou-se ingestão de água de 2,20 L/d, e que a qualidade da dieta "necessita de modificações". Exceto nos idosos em que não foi observada nenhuma associação, nos demais grupos mostrou-se que há associação positiva da ingestão de água com o IQDR, bem como com os componentes frutas totais e integrais. Estas associações foram mantidas mesmo após ajustes para variáveis de confusão. Nos adultos, observou-se que aqueles que ingeriram menos água tiveram menores pontuações para gorduras saturadas. A qualidade da dieta dos residentes de São Paulo é pior entre os que ingerem menos água.

Palavras-chave Dieta, Bebida, Inquéritos de saúde 


\section{Introdução}

Cerca de $60 \%$ do corpo humano é composto por água, cuja ingestão adequada é essencial para a estrutura e função dos tecidos, homeostase corpórea, e regulação do metabolismo ${ }^{1,2}$. A ingestão de água tem sido associada como fator positivo na prevenção de doenças crônicas não transmissíveis relacionadas à obesidade ${ }^{3}$, por auxiliar na maior saciedade $^{4,5}$ e assim viabilizar a diminuição do valor calórico diário ingerido ${ }^{6-8}$.

Pouco se conhece sobre a ingestão de água de populações, e as recomendações existentes divergem tanto por causa da variação dos fatores que são considerados para a sua definição, como idade, sexo, clima, presença de algumas doenças e uso de medicamentos, como no que é considerado consumo de água ${ }^{4,9-11}$. O Instituto de Medicina dos Estados Unidos (Institute of Medicine) - que estabelece a ingestão dietética de referência (Dietary Reference Intake - DRI $)^{9}$ - e a Agência Europeia para Segurança de Alimentos (European Food Safety Agency - EFSA) ${ }^{4}$, por exemplo, consideram em suas recomendações a ingestão de água potável, bebidas e alimentos. A Dietistas do Canadá (Dietitians of Canada), por outro lado, exclui a água proveniente dos alimentos e considera apenas a proveniente de bebidas $^{10}$.

Vale salientar que em virtude das variações que são consideradas para estabelecer as recomendações, como mencionado acima, os pontos de corte estabelecidos pelas diversas instituições acabam diferindo entre si. A DRI, por exemplo, estabelece como ponto de corte para adolescentes do sexo masculino com idade entre 14 e 18 anos o valor de 3,3 L/d 9 , já a EFSA estabelece para indivíduos com 14 anos e mais a faixa de 2,2 a $2,6 \mathrm{~L} / \mathrm{d}^{4}$, e a Dietitians of Canada adota valores somente para indivíduos com 19 anos ou mais ${ }^{10}$.

Apesar das divergências para a definição das recomendações, dados de 16.276 adultos e idosos da América Latina, Europa e Ásia apontam que a ingestão de água é insuficiente, com aproximadamente $50 \%$ dos homens e $40 \%$ das mulheres não atingindo a recomendação de água ${ }^{12}$. No entanto, apesar da baixa ingestão, estudos realizados com adultos e idosos americanos e franceses têm demonstrado que indivíduos que ingerem maiores quantidades de água tem melhor variedade da dieta, maior consumo de frutas, vegetais e grãos integrais, e menor consumo de bebidas açucaradas ${ }^{3,13,14}$. Assim, tem-se sugerido que o consumo de água pode estar associado à melhor qualidade da dieta ${ }^{6,15,16}$.

Apesar das evidências de que há baixo consumo de água de forma global, e que esta pode estar associada a qualidade da dieta, faltam estudos que demonstrem estes achados considerando amostras representativas, e, por outro lado, faltam informações sobre o consumo de água em alguns países, o que inclui o Brasil, cujas informações sobre o consumo de água são pouco ou nada explorada na literatura brasileira.

Conhecer e explorar mais esse assunto pode auxiliar no direcionamento de políticas públicas para a redução e prevenção de obesidade, por exemplo, visto que a pior qualidade da dieta está associada com esse desfecho, e o maior consumo de água parece influenciar de forma positiva na melhoria destes quadros, tendo em vista que se tem demonstrado que a ingestão de água potável auxilia na saciedade, além da hidratação, e faz com que os indivíduos busquem menos alimentos que podem fornecer calorias em excesso ${ }^{3-8}$. Assim, esse estudo se propõe, de forma pioneira no Brasil, investigar se a qualidade da dieta varia de acordo com a adequação da ingestão de água.

\section{Métodos}

O estudo foi aprovado pelo Comitê de Ética em Pesquisa da Secretaria Municipal de Saúde de São Paulo e da Faculdade de Saúde Pública da USP. O consentimento/assentimento livre e esclarecido foi obtido de todos os indivíduos e dos responsáveis, quando foi o caso, antes da coleta de qualquer informação.

\section{Delineamento e população de estudo}

Este estudo faz parte do Inquérito de Saúde de São Paulo com enfoque em Nutrição (ISA -Nutrição). O ISA-Nutrição 2015 trata-se de um estudo transversal que usa amostra complexa, estratificada e multiestágio, a fim de obter amostra representativa de residentes em domicílios fixos na área urbana da cidade de São Paulo.

A amostra $(\mathrm{N}=1.741)$ foi obtida mediante amostragem estratificada por conglomerados em dois estágios: setor censitário e domicílio. Os estratos foram compostos pelos conjuntos de setores censitários das cinco coordenadorias de saúde do município de São Paulo. No primeiro estágio foram sorteados 30 setores censitários urbanos em cada coordenadoria, totalizando 150 unidades primárias de amostragem no município. No segundo estágio foram sorteados, em cada setor, por sorteio sistemático, 18 domicílios particulares que compuseram as amostras para cada grupo de idade/sexo: adolescentes (12-19 anos), 
adultos (20-59 anos) e idosos ( $\geq 60$ anos). Mais informações podem ser lidas em Fisberg et al. ${ }^{17}$.

Dados sociodemográficos (sexo, idade, raça autodeclarada, escolaridade do chefe da família, renda familiar per capita, tabagismo, consumo de álcool e prática de atividade física), foram obtidos por meio de questionário do ISA aplicado na casa dos entrevistados. O sexo foi categorizado em variável dicotômica (masculino e feminino); a idade, em anos, foi usada de forma contínua; a raça autodeclarada foi categorizada em brancos, negros e outras raças; a escolaridade do chefe da família foi dividida em menor do que 10 anos de estudo (ensino médio incompleto) ou 10 anos ou mais (pelo menos ensino médio completo); a renda familiar per capita foi calculada pela média aritmética dos ganhos dos membros da casa, e o resultado foi categorizado em menor igual a um salário mínimo ou maior do que um salário mínimo, o tabagismo foi classificado em nunca, ex-fumante ou fumante; o consumo de álcool em consumidor e não consumidor; e a prática de atividade física, avaliada por meio do Questionário Internacional de Atividade Física (International Physical Activity Questionnaire) $)^{18}$, foi categorizada em não cumpre a recomendação e cumpre a recomendação ${ }^{19}$.

\section{Ingestão de água}

A ingestão de água total, ou seja, o consumo de água potável e da água presente nos alimentos e bebidas ${ }^{9}$ foi avaliada por meio de dois recordatórios alimentares de $24 \mathrm{~h}$ (R24h). O primeiro R24h foi coletado no domicílio por entrevistadores treinados, junto ao questionário do ISA, e o segundo foi coletado por telefone, em dia não consecutivo à primeira coleta, por estudantes de nutrição previamente treinados para a execução da coleta de dados. Ambas as coletas seguiram os procedimentos descritos no método de múltiplos passos (multiple pass method), desenvolvido pelo Departamento de Agricultura dos Estados Unidos (United States Department of Agriculture - USDA), no qual o entrevistado é conduzido por meio de cinco passos que visam manter o indivíduo interessado e engajado na entrevista, e o auxilia a recordar: 1 . Listagem rápida; 2 . Listagem de alimentos esquecidos; 3 . Tempo e ocasião das refeições; 4. Ciclo de detalhamento do consumo e 5. Revisão final ${ }^{20}$. Para complementar as informações de água, na revisão final foi feita ainda a seguinte pergunta: "Qual foi a quantidade de água consumida no dia anterior? (Registrar em medidas caseiras como copos, xícaras, garrafas)".
Após a coleta dos dados do R24h as medidas caseiras relatadas foram convertidas para unidades de peso e volume conforme descrito em Fisberg e Villar $^{21}$. Os R24h foram analisados no programa Nutrition Data System for Research (versão 2014, Nutrition Coordinating Center, Universidade de Minnesota, EUA), que incorpora os procedimentos do método de múltiplos passos.

A ingestão habitual de água total foi estimada com o auxílio do programa online multiple source method (MSM), que tem em sua plataforma incorporados métodos estatísticos baseados em três passos para chegar à estimativa de valores individuais, em que é corrigido o erro intrapessoal $^{22}$. Brevemente, a princípio, a partir dos dados inseridos o programa estima a probabilidade de ingestão do nutriente para cada indivíduo. Depois, é estimada a quantidade usual da ingestão do nutriente em dias de consumo. Por fim, os valores obtidos nos passos anteriores são multiplicados entre si para estimar a ingestão diária usual para cada indivíduo. A ingestão adequada (adequate intake - AI) de água foi usada como ponto de corte para estratificar os indivíduos, sendo estes distribuídos em: com ingestão habitual menor do que a $\mathrm{AI}(<\mathrm{AI})$, ou igual ou maior do que a $\mathrm{AI}(\geq \mathrm{AI})$ determinada para o sexo e idade correspondentes - sexo masculino: 2,4 L/d (12 a 13 anos), 3,3 L/d (14 a 18 anos) ou 3,7 L/d ( $\geq 19$ anos), sexo feminino: $2,1 \mathrm{~L} / \mathrm{d}$ (12 a 13 anos), 2,3 L/d (14 a 18 anos) ou $2,7 \mathrm{~L} / \mathrm{d}(\geq 19 \text { anos })^{9}$.

\section{Índice de qualidade da dieta}

A qualidade da dieta foi avaliada por meio do índice de qualidade da dieta revisado para a população brasileira (IQDR), que é composto por doze componentes: nove grupos de alimentos (frutas totais; frutas integrais; vegetais totais; vegetais verdes escuros, alaranjados e leguminosas; cereais totais, cereais integrais; leite e derivados; carnes, ovo e leguminosas; e óleos), dois nutrientes (gorduras saturadas e sódio) e um componente que representa a energia proveniente de gorduras sólidas, açúcar de adição e álcool ${ }^{23}$. O IQDR total é representado pela soma de todos os componentes, podendo atingir o máximo de 100 pontos. Mais informações podem ser vistas em Previdelli et al. ${ }^{23}$. A fim de classificar a população quanto ao IQDR, foram calculados os tercis do IQDR da população estudada e estabeleceu-se a seguinte classificação: "dieta inadequada" quando o IQDR for menor do que 56 pontos, "dieta necessita de modificações" quando o IQDR for entre 56 e 73 pontos, e "dieta adequada" quando este valor for superior a 73 pontos. 


\section{Análises estatísticas}

As análises estatísticas foram feitas no software Stata usando o modo survey (versão 13.0, StataCorp LP, College Station, EUA). A normalidade foi testada pelo teste de Shapiro-Wilk. Os resultados foram apresentados em média, erro padrão e distribuição em percentis. Os indivíduos foram separados de acordo com a AI de água (abaixo da AI e igual ou acima da AI) e as diferenças foram avaliadas com base nos intervalos de confiança (IC95\%), e o IQDR total e seus componentes foram comparados pelo teste de medianas para estudos com amostragem complexas Theil-Sen ${ }^{24}$. Foi considerado nível de significância de $\alpha=5 \%$.

Modelos múltiplos de regressão logística foram usados para melhor avaliar a associação da ingestão de água com o IQDR e seus componentes, considerando variáveis de possível confusão. A ingestão de água foi incluída como variável dependente e foram consideradas como variáveis independentes o IQDR ou os seus componentes, sexo, idade, raça autodeclarada, escolaridade do chefe da família, renda familiar per capita, tabagismo, consumo de álcool e prática de atividade física. Quando necessário foram feitos ajustes as variáveis independentes após análise pelo teste de Hosmer-Lemeshow, que comprova se os modelos ajustados propostos podem explicar bem o que foi observado $(\mathrm{p}>0,05)$.

\section{Resultados}

A maioria da população avaliada foi composta por mulheres (57\%), indivíduos que se autodeclarou branco (58\%), que não completou o ensino médio $(63 \%)$, com renda familiar per capita maior do que um salário mínimo (59\%), que nunca fumou $(61 \%)$, que não consome álcool (74\%) e que não cumpre a recomendação para a atividade física $(84 \%)$.

A média de consumo de água total dos indivíduos avaliados foi de 2,20 L/d. Quanto aos valores de IQDR obtidos, pode-se classificar a dieta como "necessita de modificações". Na população geral os indivíduos que consumiram menos água apresentaram menores pontuações de consumo de frutas totais, frutas integrais e sódio. Nos adolescentes, os que ingeriram menos água tiveram menores pontuações de consumo de frutas totais e integrais, vegetais totais e maior pontuação de cereais totais. Nos adultos, os que ingeriram menos água tiveram menores pontuações para frutas totais e integrais, óleos e gorduras satura- das. Nenhuma diferença foi observada entre os idosos, que pareceu ter uma ingestão uniforme independente da ingestão de água. Quanto ao IQDR total, foram observadas diferenças apenas nos adolescentes, cuja menor ingestão de água resultou em menor pontuação geral (Tabela 1).

Após ajustes por fatores de confusão (Tabela 2), a ingestão de água só não se mostrou associada ao IQDR total entre os idosos, que manteve o perfil de não associação com nenhum parâmetro do IQDR e seus componentes, sendo demonstrado nos demais grupos associação positiva da ingestão de água com o IQDR. Quanto aos componentes do IQDR, a observação de que a menor ingestão de água é associada a menor ingestão de frutas totais e integrais manteve-se para a população total, os adolescentes e adultos. Ademais, após o ajuste por fatores de confusão na população total, a associação da ingestão de água com o sódio deixou de existir, e foi observada associação inversa da ingestão de água com o consumo de cereais integrais. Nos adolescentes, após o ajuste por fatores de confusão, as demais associações além do IQDR total e das frutas tornaram-se não significativas, e entre os adultos não se pode inferir nada em relação aos óleos, uma vez que de acordo com o teste de Hosmer-Lemeshow nenhum modelo ajustado proposto explicou bem a associação, e a associação positiva com a gordura saturada foi mantida e o componente que representa a energia proveniente de gorduras sólidas, açúcar de adição e álcool também mostrou-se positivamente associado a ingestão de água.

\section{Discussão}

No presente estudo foi observado que a ingestão de frutas totais e integrais na população total, nos adolescentes e nos adultos varia com a adequação da ingestão de água, bem como a ingestão de cereais totais nos adolescentes, e a ingestão de óleos e gorduras saturadas nos adultos. Os idosos parecem não variar a qualidade da dieta de acordo com a ingestão de água.

Discute-se que diversos fatores estão relacionados ao consumo de água total ${ }^{4,9,25}$, como os considerados pelas DRI para o estabelecimento da AI, que são: idade, sexo, nível de atividade física, clima, tipo de atividade física, altitude do local, presença de doenças como diabetes mellitus, fibrose cística e uso de medicamentos diuréticos ou outros que possam interferir no balanço hídrico ${ }^{9}$, assim como outros fatores, como a qualidade da dieta e seus componentes ${ }^{3,25}$. No entanto, 


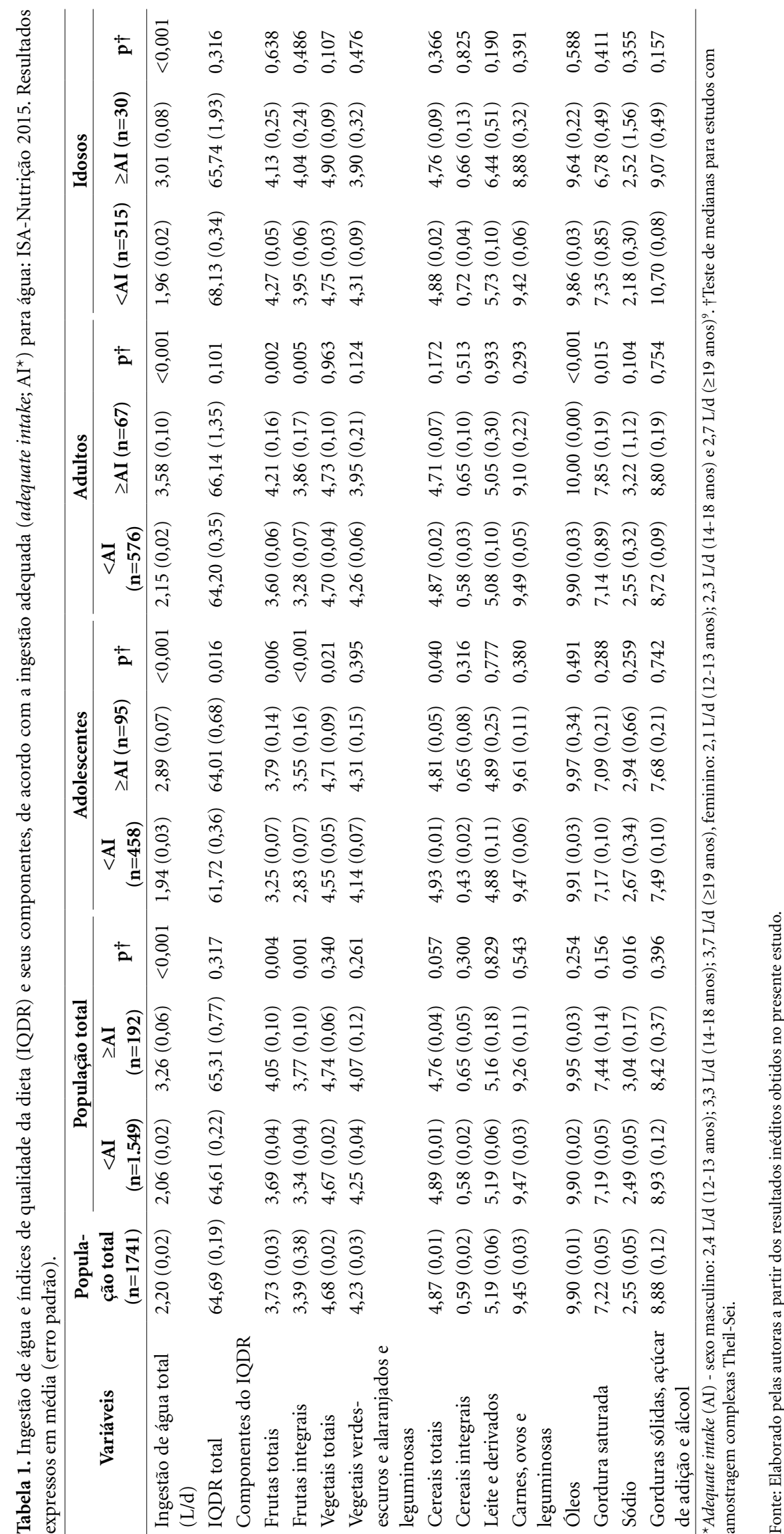


Tabela 2. Associação da ingestão de água com os índices de qualidade da dieta (IQDR) e seus componentes, considerando possíveis fatores de confusão: ISA-Nutrição 2015. OR razão de chances e IC95\% intervalo de confiança de $95 \%$.

\begin{tabular}{|c|c|c|c|c|c|c|c|c|}
\hline \multirow{3}{*}{ Variáveis } & \multicolumn{8}{|c|}{ Ingestão de água* } \\
\hline & \multicolumn{2}{|c|}{ População total } & \multicolumn{2}{|c|}{ Adolescentes } & \multicolumn{2}{|c|}{ Adultos } & \multicolumn{2}{|c|}{ Idosos } \\
\hline & OR & IC95\% & OR & IC95\% & OR & IC95\% & OR & IC95\% \\
\hline IQDR total & 1,04 & 1,$02 ; 1,08$ & $1,05 \ddagger$ & 1,$00 ; 1,10$ & $1,07 \ddagger$ & 1,$03 ; 1,11$ & $0,96+\dagger$ & 0,$90 ; 1,02$ \\
\hline Frutas totais & 1,46 & 1,$23 ; 1,72$ & 1,30 & 1,$05 ; 1,61$ & 1,449 & 1,$13 ; 1,84$ & 0,9399 & 0,$69 ; 1,26$ \\
\hline Frutas integrais & $1,30 \dagger$ & 1,$13 ; 1,51$ & 1,27 & 1,$04 ; 1,55$ & 1,42 & 1,$14 ; 1,76$ & 1,09 扭 & 0,$81 ; 1,48$ \\
\hline Vegetais totais & $1,20 \ddagger$ & 0,$96 ; 1,50$ & 1,15 & 0,$88 ; 1,50$ & 1,22 & 0,$85 ; 1,75$ & 1,4599 & 0,$74 ; 2,80$ \\
\hline $\begin{array}{l}\text { Vegetais verdes-escuros e } \\
\text { alaranjados e leguminosas }\end{array}$ & 0,98 & 0,$83 ; 1,14$ & $1,13 \ddagger$ & 0,$93 ; 1,38$ & $0,96 \dagger$ & 0,$79 ; 1,18$ & $0,84 \# \#$ & 0,$62 ; 1,13$ \\
\hline Cereais totais & 0,68 & 0,$50 ; 0,90$ & $0,53 \ddagger$ & 0,$23 ; 1,21$ & 0,72 & 0,$53 ; 0,98$ & 0,6099 & 0,$32 ; 1,12$ \\
\hline Cereais integrais & $1,17 \ddagger$ & 0,$88 ; 1,56$ & 1,14 & 0,$76 ; 1,73$ & $1,29 \#$ & 0,$85 ; 1,96$ & 0,97 抹 & 0,$61 ; 1,52$ \\
\hline Leite e derivados & 1,02 & 0,$93 ; 1,11$ & 1,02 & 0,$89 ; 1,15$ & 0,99 & 0,$88 ; 1,12$ & 1,1699 & 0,$93 ; 1,44$ \\
\hline Carnes, ovos e leguminosas & 0,97 & 0,$84 ; 1,11$ & $1,23 \ddagger$ & 0,$94 ; 1,61$ & 0,96 & 0,$82 ; 1,14$ & 0,8099 & 0,$64 ; 1,00$ \\
\hline Óleos & 1,18 & 0,$73 ; 1,92$ & 1,34 & 0,$81 ; 2,22$ & - & - & $0,72^{\star * *}$ & 0,$46 ; 1,13$ \\
\hline Gordura saturada & 1,14 & 0,$99 ; 1,30$ & 1,03 & 0,$88 ; 1,20$ & $1,31^{\star *}$ & 1,$10 ; 1,57$ & $0,90^{\star * *}$ & 0,$72 ; 1,12$ \\
\hline Sódio & $1,02 \ddagger$ & 0,$93 ; 1,12$ & 0,97 & 0,$83 ; 1,13$ & 1,05 & 0,$92 ; 1,19$ & - & - \\
\hline $\begin{array}{l}\text { Gorduras sólidas, açúcar de } \\
\text { adição e álcool }\end{array}$ & 1,03 & 0,$99 ; 1,06$ & $1,03 \ddagger$ & 0,$97 ; 1,10$ & 1,06 & 1,$00 ; 1,11$ & $0,93 \# \#$ & 0,$85 ; 1,03$ \\
\hline
\end{tabular}

Os resultados foram ajustados por sexo, idade, raça declarada, escolaridade do chefe da família, renda familiar per capita, tabagismo, consumo de álcool e prática de atividade física. As caselas em branco significa que nenhum modelo ajustado passou pelo teste de Hosmer-Lemeshow $(>0,05)$. ${ }^{\star}$ Os indivíduos foram classificados quanto à adequação ou inadequação da ingestão de água de acordo com a adequate intake correspondente ao seu sexo e idade. †Os resultados foram ajustados por sexo, idade, raça declarada, escolaridade do chefe da família, renda familiar per capita, tabagismo e prática de atividade física. ‡Os resultados foram ajustados por sexo, idade, escolaridade do chefe da família, renda familiar per capita, tabagismo, consumo de álcool e prática de atividade física. Os resultados foram ajustados por sexo, idade e prática de atividade física. \#Os resultados foram ajustados por sexo, idade, raça declarada, renda familiar per capita, tabagismo, consumo de álcool e prática de atividade física. ${ }^{* *}$ Os resultados foram ajustados por sexo, idade, renda familiar per capita e prática de atividade física. ††Os resultados foram ajustados por idade e consumo de álcool. \$¥Os resultados foram ajustados por idade e prática de atividade física. 990 resultados foram ajustados por idade. \#\#Os resultados foram ajustados por idade, tabagismo e prática de atividade física. ${ }^{* * *}$ Os resultados foram ajustados por idade, tabagismo, consumo de álcool e prática de atividade física.

Fonte: Elaborado pelas autoras a partir dos resultados inéditos obtidos no presente estudo.

até o momento não se chegou a um consenso, o que dificulta a comparação dos resultados de forma global entre os estudos, e, de alguma maneira, o próprio estabelecimento de valores de recomendações média estimadas (estimated average requirements) para água, tendo-se disponível, até o momento, valores de AI que não nos permite estimar a prevalência de inadequação. No Brasil, as orientações do Instituto de Medicina dos Estados Unidos da América embasam as orientações sobre consumo de água feitas pelo Guia Alimentar para a População Brasileira ${ }^{26}$.

Apesar desta limitação, considerando a AI como ponto de corte, percebe-se que há indícios de que há baixa probabilidade de adequação da ingestão de água. Os idosos parecem ter menor probabilidade de adequação em relação aos demais grupos. Este fato mostra-se mais preocupante, pois como as crianças, este grupo tem maior risco para a desidratação, dadas as con- dições fisiológicas comuns à idade ${ }^{9}$. É relevante destacar que embora exista uma boa correlação do relato de consumo de idosos e o consumo real, é preciso reconhecer que a perda de função cognitiva e a perda de memória decorrente da idade podem interferir no relato e serem fatores de viés no relato do consumo de água nos idosos, especialmente.

Têm-se sugerido que a ingestão de água está associada a qualidade da dieta de forma global, pois a menor ingestão de água estaria relacionada a piores padrões alimentares, com menor presença de frutas e verduras ${ }^{3,25}$, que são alimentos fontes de água e cuja maior ingestão auxiliaria na maior ingestão de água ${ }^{9,27}$. No presente estudo a possível baixa ingestão de água, por um lado, não demonstrou ser um fator para pior qualidade da dieta entre os idosos, mas por outro, mostrou-se relevante principalmente entre os adolescentes, cuja menor ingestão de água afetou inclusive o 
índice total (IQDR). Ademais, pontualmente quanto as frutas, tanto totais como integrais, além do IQDR para os adolescentes, o presente estudo confirmou que os indivíduos que ingerem menos água têm menor ingestão especialmente de frutas. O mesmo foi observado em estudo realizado nos Estados Unidos, que verificou a influência do consumo de água no padrão dietético de indivíduos com 18 anos e mais, e verificou que os não consumidores de água tiveram menor consumo de frutas e vegetais ${ }^{13}$.

Por outro lado, a observação no presente estudo de que os adultos que ingeriram menos água tiveram menores índices para os componentes óleos e gorduras saturadas, e na população geral tiveram menor índice para sódio demonstra preocupação e curiosidade, haja visto que o menor índice indica, para estes componentes em específico, que há maior consumo de alimentos fontes de gorduras e sódio e, pelo nosso conhecimento, não há estudos que tenham tentado avaliar o consumo de água nesta vertente. Tais observações podem estar relacionadas a preparações culinárias como sopas, feijões e caldos, que possuem grande quantidade de água e podem contribuir tanto para o maior consumo de gorduras como de sódio. A análise de contribuintes de água nesta população pode auxiliar nesta avaliação, e estes dados serão apresentados em trabalhos futuros.

Apesar de nenhuma diferença ter sido observada no presente estudo para o componente gorduras sólidas, açúcar de adição e álcool, estudos têm demonstrado que o consumo de bebidas açucaradas - uma das fontes de açúcar de adição - tem se mostrado associado a pior qualidade da dieta ${ }^{14}$, e quando estas bebidas açucaradas são substituídas por água, se observa melhora da qualidade da dieta, como demonstrado em estudo realizado na comunidade Lower Delta Misissip $i^{7}$, o que serve de alerta para a importância da água na redução de bebidas açucaradas.

Como limitações deste estudo, há de se reconhecer que os métodos de avaliação de ingestão alimentar são suscetíveis a erros por omissões de alimentos, erros de estimativa de porções e a variância intrapessoal dos participantes. No entanto, para minimizar este viés o R24h foi conduzido por entrevistadores treinados usando o método de múltiplos passos que diminui a omissão de alimentos esquecidos e padronizam o nível de detalhamento dos alimentos. Além disso, utilizou-se o programa MSM no intuito de minimizar a variância intrapessoal, que embora ele não tenha o poder de remover completamente todos os erros, é uma ferramenta útil e gratuita para minimizar este problema, e gerar dados que sejam o mais representativo possível da dieta habitual. Vale ressaltar ainda que o MSM não permite que seja considerado o desenho amostral do estudo no ajuste da variabilidade, sendo considerada uma amostra aleatória simples. No entanto, o desenho amostral do estudo foi considerado em todas as análises estatísticas realizadas, por meio do uso do comando survey do Stata, o que inclui os valores individuais de consumo de água ajustados pelo MSM.

Finalmente, em relação à água, é necessário destacar a falta de precisão dos entrevistados para quantificar sua ingestão diária, e para isto foi utilizada uma pergunta específica no questionário para ajudar o participante a quantificar com maior precisão sua ingestão de água. Por outro lado, é necessário reconhecer ainda que os valores de AI usados para a ingestão de água foram estabelecidos para a população norte americana e canadense, e que os valores para brasileiros ou residentes no Brasil podem diferir, principalmente considerando o clima tropical, mas diante da inexistência de valores de recomendações para ingestão de água no Brasil, e considerando ainda que os valores de ingestão adequada normalmente se baseiam nas DRI, a prática do uso das DRI acaba sendo uma das melhores opções.

Cabe ressaltar ainda que a maioria dos estudos não se preocupa em questionar sobre o consumo de água potável, o que pode ser um viés dependendo do objetivo do estudo, e até o momento os estudos que buscaram validar qual o melhor método de avaliação do consumo alimentar para estimar o consumo do componente alimentar água (água total) não conseguiram chegar a uma conclusão de qual seria o melhor, mesmo quando eram feitos questionários de consumo para este fim. Desta forma, cabe ao pesquisador definir o método mais viável para o seu estudo e tomar as medidas possíveis e cabíveis para reduzir os erros inerentes a cada método ${ }^{28-32}$.

\section{Conclusão}

A qualidade da dieta é pior entre os que ingerem menos água. Estudar os fatores que interferem no consumo de água, como fatores sociodemográficos e de estilo de vida podem auxiliar no melhor direcionamento de como intervir nesta população a fim de melhorar o seu consumo de água e qualidade da dieta, bem como auxiliar no planejamento de políticas públicas de saúde. 


\section{Colaboradores}

I Lucchesi contribuiu na análise e interpretação dos dados e redação do artigo. RM Fisberg contribuiu na concepção e delineamento do estudo e revisou criticamente o artigo. $\mathrm{CH}$ Sales contribuiu na concepção e delineamento do estudo, análise e interpretação de dados e revisou criticamente o artigo. Todos os autores aprovaram a versão final apresentada e são responsáveis pela garantia da exatidão e da integridade de qualquer parte do artigo.

\section{Agradecimentos}

Agradecemos a equipe do Inquérito de Saúde de São Paulo (ISA-Capital) 2015, em especial aos professores Dr. Chester Luiz Galvão Cesar, Dr. Moises Goldbaum, pesquisadores principais do estudo ISA-Capital, e a estatística Dra. Maria Cecília Goy Porto Alves, responsável pela amostragem do estudo. Agradecemos ainda aos integrantes do grupo de avaliação do consumo alimentar (GAC-USP), especialmente a MSc. Mariane de Mello Fontanelli, e a todos os voluntários que aceitaram participar da pesquisa.

\section{Financiamento}

Este trabalho foi financiado pela Secretaria $\mathrm{Mu}-$ nicipal de Saúde de São Paulo, Fundação de Amparo à Pesquisa do Estado de São Paulo, Conselho Nacional de Desenvolvimento Científico e Tecnológico e a Coordenação de Aperfeiçoamento de Pessoal de Nível Superior.

\section{Referências}

1. Ferreira-Pêgo A, Guelinckx I, Moreno LA, Kavouras SA, Gandy J, Martinez H, Bardosono S, Abdollahi M, Nasseri E, Jarosz A, Babio N, Salas-Salvadó J. Total fluid intake and it's determinants: cross-sectional surveys among adults in 13 countries worldwide. Eur J Nutr 2015; 54(Supl. 2):35-43.

2. Otten JJ, Hellwig JP, Meyers LD, editores. Water (Parte II). In: Otten JJ, Hellwig JP, Meyers LD, editors. DRI: dietary reference intakes the essential guide to nutrient requirements. Washington, D.C.: The National Academies Press; 2006. p.156-166.

3. Gazan R, Sondey J, Maillot M, Guelinckx I, Lluch A. Drinking water intake is associated with higher diet quality among French adults. Nutrients 2016; 8(11):689.

4. European Food Safety Authority (EFSA). EFSA Panel on Dietetic Products, Nutrition, and Allergies (NDA). EFSA J 2010; 8(3):1459-1507.

5. Malik VS, Popkin BM, Bray GA, Després JP, Hu FB. Sugar sweetened beverages, obesity, type 2 diabetes and cardiovascular disease risk. Circulation 2010; 121(11):1356-1364.

6. Stookey FD, Constant F, Gardner CD, Popkin BM. Replacing sweetened caloric beverages with drinking water is associated with lower energy intake. Obesity 2007; 15(12):3013-3022.

7. Thomson JL, Tussing-Humphreys LM, Onufrak SJ, Connell CL, Zoellner JM, Bogle ML, Yadrick K. Simulated reductions in consumption of sugar-sweetened beverages improves diet quality in Lower Mississippi Delta adults. Food Nutr Res 2011; 55:e7304.

8. Dennis EA, Dengo AL, Comber DL, Flack KD, Savla J, Davy KP, Davy BM. Water consumption increases weight loss during a hypocaloric diet intervention in middle-aged and older adults. Obesity 2010; 18(2):300-307.

9. Institute of Medicine (U.S.). Water. In: Institute of Medicine (U.S.). Dietary reference intakes for water, potassium, sodium, chloride, and sulfate. Washington D.C.: The National Academies Press; 2005. p.73-166.

10. Dietitians of Canada. Guidelines for drinking fluids to stay hydrated [Internet]. Toronto: Dietitians of Canada; 2018 [acessado 2018 jan 10]. Disponível em: http://www.unlockfood.ca/en/Articles/Water/Facts-on-Fluids-How-to-Stay-Hydrated.aspx.

11. Elmadfa I, Meyer AL. Patter of drinking and eating across the Europeans Union: implications for hydration status. Nutr Rev 2015; 73(Supl. 2):141-147.

12. Iglesia I, Santaliestra-Pasías AM, Bel-Serrat S, SadallaCollese T, Miguel-Berges ML, Moreno LA. Fluid consumption, total water intake and first morning urine osmolality in Spanish adolescents from Zaragoza: data from the HELENA study. Eur J Clin Nutr 2016; 70(5):541-547.

13. Popkin BM, Barclav DV, Nielsen SJ. Water and food consumption patterns of U.S. adults from 1999 to 2001. Obes Res 2005; 13(12):2146-2152. 
14. Kant AK, Graubard BI, Atchison EA. Intakes of plain water, moisture in foods and beverages, and total water in the adult US population-nutritional, meal pattern and body weight correlates: National Health and Nutrition Examination Surveys 1999-2006. Am J Clin Nutr 2009; 90(3):655-663.

15. Edelenyi FS, Druesne-Pecollo N, Arnault N, Gonzãlez R, Buscail C, Galan P. Characteristics of beverage consumption habits among a large sample of French adults: associations with total water and energy intakes. Nutrients 2016; 8(10):627.

16. Kant AK, Graubard BI. Contributors of water intake in US children and adolescents: associations with dietary and meal characteristics-National Health and Nutrition Examination Survey 2005-2006. Am J Clin Nutr 2010; 92(4):887-896.

17. Fisberg RM, Sales CH, Fontanelli MM, Pereira JL, Alves MCGP, Escuder MML, César CLG, Goldbaum M. 2015 Health Survey of São Paulo with focus in nutrition: rationale, design, and procedures. Nutrients 2018; 10(2):169.

18. Matsudo S, Araújo T, Matsudo V, Andrade D, Andrade E, Oliveira LC, Braggion G. Questionário Internacional de Atividade Física (IPAQ): estudo de validade e reprodutibilidade no Brasil. Rev Bras Ativ Fis Saude 2001; 6(2):5-18.

19. World Health Organization (WHO). Global recommendations on physical activity for health. Genebra: WHO; 2010.

20. Rarper N, Perloff B, Ingwersen L, Steinfeldt L, Anand J. An overview of USDA's Dietary Intake Data System. J Food Compos Anal 2004; 17(3-4):545-555.

21. Fisberg RM, Villar BS. Manual de receitas e medidas caseiras para cálculo de inquéritos alimentares. São Paulo: Signus; 2002.

22. German Institute of Human Nutrition Potsdam -Rehbrücke (DIfE). The Multiple Source Method (MSM) [Internet]. Versão 1.0.1. Potsdam: DIfE; 2012 [acessado 2018 jan 10]. Disponível em: https://nugo. dife.de/msm.

23. Previdelli AN, Andrade SC, Pires MMP, Ferreira SRG, Fisberg RM, Marchioni DM. Índice de qualidade da dieta revisado para população brasileira. Rev Saude Publica 2011; 45(4):794-798.

24. Roger N. Confidence intervals for rank statistics: percentile slopes, differences, and ratios. Stata J 2006; 6(4):497-520.

25. Illescas-Zarate D, Espinosa-Montero J, Flores M, Barquera S. Plain water consumption is associated with lower intake of caloric beverage: cross-sectional study in Mexican adults with low socioeconomic status. BMC Public Health 2015; 15:405.

26. Brasil. Ministério da Saúde (MS). Guia alimentar para a população brasileira. 2a ed. Brasília: MS; 2014.

27. Montenegro-Bethancourt G, Johner SA, Remer T. Contribution of fruit and vegetable intake to hydration status in schoolchildren. Am J Clin Nutr 2013; 98(4):1103-1112.
28. Hedrick VE, Comber DL, Estabrooks PA, Savla J, Davy $\mathrm{BM}$. The beverage intake questionnaire: determining initial validity and reliability. J Am Diet Assoc 2010; 110(8):1227-1232.

29. Malisova O, Bountziouka V, Panagiotakos DB, Zampelas A, Kapsokefalou M. The water balance questionnaire: design, reliability and validity of a questionnaire to evaluate water balance in the general population. Int J Food Sci Nutri 2012; 63(2):138-144.

30. Nissensohn M, Lopez-Ufano M, Castro-Quezada I, Serra-Majem L. Assessment of beverage intake and hydration status. Nutr Hosp 2015; 31(Supl. 3):62-69.

31. Nissensohn M, Ruano C, Serra-Majem L. Validation of beverage intake methods vs. hydration biomarkers: a short review. Nutr Hosp 2013; 28(6):1815-1819.

32. Gandy J. Water intake: validity of population assessment and recommendations. Eur J Nutr 2015; 54(Supl. 2):11-16.

Artigo apresentado em 11/09/2018

Aprovado em 08/11/2019

Versão final apresentada em 10/11/2019

Editores-chefes: Romeu Gomes, Antônio Augusto Moura da Silva 
